\title{
Gasificación de lodos procedentes de Estaciones Depuradoras de Aguas Residuales. Estudio del proceso para la limpieza y mejora de las propiedades del gas producto
}

\author{
N. Gil Lalaguna, J.L. Sánchez Cebrián, M.B. Murillo Esteban \\ Grupo de Procesos Termoquímicos (GPT) \\ Instituto de Investigación en Ingeniería de Aragón (I3A) \\ Universidad de Zaragoza, Mariano Esquillor s/n, 50018, Zaragoza, Spain. \\ Tel. +34-976762224, e-mail: noemigil@unizar.es
}

\begin{abstract}
La valorización y gestión de los fangos generados en las Estaciones Depuradoras de Aguas Residuales (EDAR) es un asunto de interés en la actualidad debido al aumento del volumen generado de dicho residuo y a las restricciones impuestas sobre las vías de gestión habituales. La gasificación de estos lodos para la obtención de un gas de síntesis y/o con buenas propiedades combustibles es una posible alternativa ya que permitiría la valorización de este residuo. Sin embargo, la formación de alquitranes (hidrocarburos condensables) durante la gasificación supone ciertas limitaciones para la aplicabilidad del gas. Entre las medidas existentes para la reducción del contenido de alquitranes se encuentra una correcta selección de los parámetros de operación en el propio gasificador. Por ello, la primera parte de este trabajo se centra en el estudio de la influencia de variables como la temperatura, el tipo de agente gasificante utilizado (aire o mezclas de aire y vapor de agua) o la cantidad de agente gasificante utilizado por kilogramo de lodo procesado en el lecho fluidizado. Una vez optimizadas las condiciones de operación del gasificador, la segunda parte del trabajo consiste en la aplicación de catalizadores para el reformado de los alquitranes en fase vapor. Con el objetivo de evaluar la actividad de distintos tipos de catalizadores se está trabajando en una planta de lecho fijo, en la que se alimenta un gas con una composición similar al gas de gasificación y se utilizan compuestos modelo para simular la presencia de alquitranes en el gas. Los catalizadores que mejores resultados ofrezcan serán utilizados en la limpieza del gas real que se obtiene en la gasificación de los lodos, incorporando un lecho catalítico aguas abajo del gasificador.
\end{abstract}

